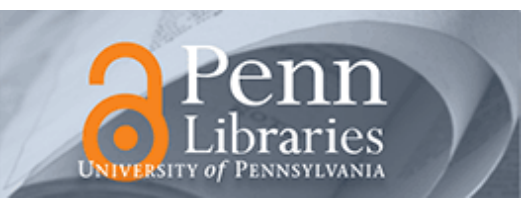

University of Pennsylvania

ScholarlyCommons

$12-9-2015$

\title{
Exploring the Relationship of Patient and Informal Caregiver Characteristics with Heart Failure Self-Care Using the Actor- Partner Interdependence Model: Implications for Outpatient Palliative Care
}

Harleah G. Buck

Jacqueline Mogle

Barbara Riegel

University of Pennsylvania, briegel@nursing.upenn.edu

Susan McMillan

Marie A. Bakitas

Follow this and additional works at: https://repository.upenn.edu/nrs

Part of the Cardiology Commons, Cardiovascular Diseases Commons, Circulatory and Respiratory Physiology Commons, Medical Humanities Commons, Nursing Commons, Palliative Care Commons, and the Preventive Medicine Commons

\section{Recommended Citation}

Buck, H. G., Mogle, J., Riegel, B., McMillan, S., \& Bakitas, M. A. (2015). Exploring the Relationship of Patient and Informal Caregiver Characteristics with Heart Failure Self-Care Using the Actor-Partner Interdependence Model: Implications for Outpatient Palliative Care. Journal of Palliative Medicine, 18 (12), 1026-1032. http://dx.doi.org/10.1089/jpm.2015.0086 


\title{
Exploring the Relationship of Patient and Informal Caregiver Characteristics with Heart Failure Self-Care Using the Actor-Partner Interdependence Model: Implications for Outpatient Palliative Care
}

\begin{abstract}
Background: The convergence of prevalence, cost, symptom experience, community setting, and informal caregiving in heart failure (HF) has profound implications for outpatient palliative care. The majority of HF patients depend on informal caregiver's assistance. Dyadic (patients and caregiver) characteristics can complicate this assistance. Yet relatively little is known concerning dyadic characteristics' impact on selfcare. HF self-care involves routine, daily treatment adherence and symptom monitoring (self-care maintenance), and symptom response (self-care management).

Objective: Describe the dyadic characteristics of mood and perception of the relationship in HF patients and caregivers, then explore the relationship of the characteristics with self-care.

Design: Prospective, cross sectional study of hospitalized HF patients in mixed dyads (spousal/adult child/relative) analyzed using Actor-Partner Interdependence Model (APIM) techniques.

Measurements: Mood was measured by the Brief Symptom Inventory and Patient Health Questionnaire, perception of the relationship by the Dyadic Adjustment Scale, and self-care by the Self-care in Heart Failure Index.

Results: In 40 dyads the average patient was a 71 year old male $(n=30)$; caregiver was a 59 years old female $(n=26)$. Overall self-care scores were consistently low. Patient depression scores were significantly greater than caregivers $(p=.0055)$. Greater caregiver anxiety were associated with lower caregiver maintenance scores $(p<.0001)$ but greater caregiver depression were associated with lower patient maintenance scores $(p<.0001)$. While patient and caregiver's perception of the relationship was associated with their self-care, more importantly, caregiver's perception of the relationship was associated with their confidence to engage in the patient's self-care $(p=.003)$.
\end{abstract}

Discussion/Conclusion: This study suggests that caregivers, often unacknowledged or unmeasured, impact patient's day to day HF self-care. Palliative care clinicians need to talk to dyads with a history of poor self-care about their relationship.

\section{Disciplines}

Cardiology | Cardiovascular Diseases | Circulatory and Respiratory Physiology | Medical Humanities | Medicine and Health Sciences | Nursing | Palliative Care | Preventive Medicine 
Title: Exploring the Relationship of Patient and Informal Caregiver Characteristics with Heart Failure Self-care Using the Actor-Partner Interdependence Model: Implications for Outpatient Palliative Care

\title{
Corresponding author:
}

Harleah G. Buck, PhD, RN, CHPN, FPCN

College of Nursing

The Pennsylvania State University

201 Health and Human Development East

University Park, PA 16802

Phone: $\underline{814-863-3495}$

Fax: $814-865-3779$

Email: hgb2@psu.edu

\section{Co-authors:}

Jacqueline Mogle, PhD

Barbara Riegel, PhD, RN, FAAN, FAHA

Susan McMillan, PhD, ARNP, FAAN

Marie Bakitis, DNSc, APRN, AOCN, ACHPN, FAAN

\begin{abstract}
Background: The majority of heart failure (HF) patients depend on informal caregiver's assistance. Dyadic (patients and caregiver) characteristics complicate this assistance. Relatively little is known concerning dyadic characteristics' impact on self-care. HF self-care involves routine, daily treatment adherence and symptom monitoring (self-care maintenance), and symptom response (self-care management).

Objective: Describe the dyadic characteristics of mood and perception of the relationship in HF patients and caregivers, then explore the relationship of the characteristics with self-care.

Design: Prospective, cross sectional study of hospitalized HF patients in mixed dyads (spousal/adult child/relative) analyzed using Actor-Partner Interdependence Model (APIM) techniques.

Measurements: Mood was measured by the Brief Symptom Inventory and Patient Health Questionnaire; perception of the relationship by the Dyadic Adjustment Scale; and self-care by the Self-care in Heart Failure Index.
\end{abstract}


Results: In 41 dyads the average patient was a 71 year old male $(n=30)$; caregiver was a 59 years old female $(n=26)$. Overall self-care scores were consistently low. Patient depression scores were significantly greater than caregivers $(p=.0055)$. Greater caregiver anxiety were associated with lower caregiver maintenance scores $(p<.0001)$ but greater caregiver depression were associated with lower patient maintenance scores $(p<.0001)$. While patient and caregiver's perception of the relationship was associated with their self-care, more importantly, caregiver's perception of the relationship was associated with their confidence to engage in the patient's self-care $(p=.003)$.

Discussion/Conclusion: This study suggests that caregivers, often unacknowledged or unmeasured, impact patient's day to day HF self-care. Clinicians need to talk to dyads with a history of poor self-care about their relationship. 


\section{Introduction}

Advanced heart failure (HF) is one of the most prevalent and costly chronic illnesses with a symptom experience comparable to cancer. ${ }^{1-3}$ Much of this cost is driven by emergency care secondary to symptom exacerbation. ${ }^{4,5}$ The majority of HF patients remain in the community through the end of their lives and depend on informal caregivers to assist with managing their HF symptoms. ${ }^{6,7}$ The convergence of prevalence, cost, symptom experience, community setting, and informal caregiving has profound implications for outpatient palliative care.

HF self-care involves routine, daily treatment adherence and symptom monitoring (self-care maintenance), and symptom response (self-care management). ${ }^{8}$ Self-care confidence or self-efficacy is known to facilitate self-care. ${ }^{9}$ Recent studies suggest that patients' and caregivers' engagement in self-care impacts their own and their care partner's quality of life ${ }^{10}$ and that patients engage less and caregivers engage more as $\mathrm{HF}$ progresses and the patient becomes sicker. ${ }^{11}$ But what factors impact the patient and caregiver's decision to engage or how fully to engage in self-care is currently poorly understood.

Although many characteristics may serve to differentiate dyads and potentially contribute to HF dyad's successful or unsuccessful HF self-care, we examined actor (patient) and partner (caregiver) effects ${ }^{12}$ of mood and perception on HF self-care as both are modifiable and therefore amenable to intervention. Mood (anxiety and depression) $)^{13,14}$ and perceptions (of symptoms and the relationship) ${ }^{15,16}$ are known to influence one's own mood and perception as well as one's partner's, but less is known about whether these characteristics influence engagement in HF self-care. There is a body of work illustrating that relational satisfaction or the person's perception of their relationship is a contextual element in chronic illness management. ${ }^{17}$ But, again, the link between patients' or caregivers' perception of the relationship and patients' self-care has not been explored in HF dyads. The geriatric literature suggests that the quality of spousal relationships (one common type of dyadic relationship) moderates the association between the effects of symptoms on both partners ${ }^{18}$ but once again, this has not been examined in HF. Tantalizing 
glimpses are seen into the importance of dyadic interaction in HF from a study that found that spousal distress predicted increases in patients' symptoms and poorer general health. ${ }^{14}$ A series of recent palliative care papers ${ }^{19-21}$ support the impact of $\mathrm{HF}$ on the dyad ${ }^{19}$; the importance of dyadic congruence in improving patient outcomes in $\mathrm{HF}^{20}$; and that caregivers' perception of the relationship is associated with their perception of benefit or burden in their role. ${ }^{21}$ But unexamined is the important link of the dyad to self-care outcomes. The specific aim of this study was to first, describe the dyadic characteristics of mood and perception of the relationship in HF patients and caregivers, then explore the relationship of these dyadic characteristics with HF self-care.

Methods

Study design

This was a small, prospective study using cross sectional data. The Actor Partner Interdependence model $^{22}$ (Figure 1) provided the conceptual and analytic framework. This model posits that patient and caregiver characteristics are interdependent. The model accounts for this lack of independence by modeling the effect of the individual on their own outcomes as actor effects and the effects on the partner as partner effects. This project was not powered to test the model per se but rather to generate information on the participants, variables, and strength of the relationships between the variables.

\section{Setting and Sample}

Forty one Stage $\mathrm{C}$ or $\mathrm{D}^{1} \mathrm{HF}$ patient and caregiver dyads were recruited from a community health system and an academic medical center in the Northeastern United States. Institutional Review Board approval was obtained from both institutions and informed consent was obtained from both patient and caregiver. Inclusion criteria: patient and caregivers were both adults, 18 years of age or older, patient had a documented HF diagnosis in the medical record, and patient and caregiver willing and able to participate. 
Patients were excluded it they had received a heart transplantation or a left ventricular assist device. These exclusion criteria were devised to assure the homogeneity of the caregiving experience. ${ }^{23}$ Trained research assistants screened daily hospital admission records for patients admitted with a HF diagnosis in the previous 24 hours who met the inclusion criteria. This time was selected to allow for recent recall of symptom management. Patients were approached, the study was explained, consent was obtained and questionnaire packets were completed in one visit. If a caregiver was not present at the visit, a packet was sent to the caregiver for completion.

\section{Measures}

Depression was measured by Patient Health Questionnaire-9 (PHQ-9). Responses range from 0 ("not at all") to 3 ("nearly every day") for a total score of 1 to 27 with a score of 10 used as a cut point for major depression. ${ }^{24}$ The PHQ-9 is reported to have a sensitivity of $88 \%$ and specificity of $88 \%$ for detecting a major depression. ${ }^{24}$ In this sample the coefficient alpha for the patient was .80 and for the caregiver was .91 .

Anxiety was measured by the Brief Symptom Inventory (BSI). ${ }^{25}$ The anxiety subscale consists of 6 statements rated on a scale of 0 ("not at all") to 4 ("extremely"). The average of the 6 items comprises the total score with a score of .35 or higher used as a cut point for anxiety. Reliability in the current sample was considered excellent (patient $\alpha=.82$, caregiver $\alpha=.91$ ).

Perception of the relationship was assessed using the Dyadic Adjustment Scale short form (DAS-7) developed by Sharpley and Rogers ${ }^{26}$ from the longer version. ${ }^{27}$ The DAS-7 consists of 6 statements about a person's perception of their degree of agreement on relational factors such as shared philosophy, goals and time spent together. Participants are required to rate items on a scale from 0 ("always disagree") to 5 ("always agree"). A seventh item asks their perception of degree of happiness in the relationship on a scale from 0 ("extremely unhappy") to 6 ("perfect"). All items are summed to create a total score. In the current sample, reliability was acceptable for both members of the dyad (patient $\alpha=.70$, caregiver $\alpha=.78$ ). 
Self-care maintenance, management and confidence for the patient was measured by the Self-care of Heart Failure Index which includes a scale for each of the constructs. ${ }^{28}$ The 10 item maintenance scale focuses on treatment adherence, such as following medication, diet, and exercise guidelines, and symptom monitoring on a 1 ("never or rarely") to 4 ("always or daily") point scale. The 6 item management scale assesses the ability of the patient to recognize symptom occurrence, implement a meaningful treatment, and evaluate the outcome of that treatment choice on a 1-4 point scale. The 6 item confidence scale assesses the perception of HF self-care self-efficacy on a 1-4 point scale. For all scales higher responses reflect better selfcare maintenance, management, and greater perceptions of self-care self-efficacy. Internal reliability and construct validity have been shown in multiple studies. ${ }^{8,29,30}$ Maintenance, management and self-care selfefficacy for the caregiver was measured by an adaptation of the patient scales. This was developed in collaboration with other HF researchers and a similar caregiver adaptation has shown comparable psychometric properties with the patient instrument. ${ }^{31,32}$

Patient and caregiver sociodemographic data was by self-report, including age, gender, race/ethnicity, education level, and income.

\section{Data Analysis}

The current analyses used actor partner interdependence models ${ }^{33}$ (APIMs) implemented using a structural equation modeling framework in MPLUS. ${ }^{34}$ These models allow a researcher to simultaneously calculate the effects of the patient's experiences (i.e., mood state and perceptions of the relationship) on their own self-care maintenance, management, or self-efficacy and the effect of their experiences on the caregiver's ratings of the same outcome and vice versa. These models also account for the intercorrelation among partners for both the predictors and outcomes. Models in the current analysis were considered justidentified because all possible relationships among variables were specified. Just-identified models will always have perfect model $\mathrm{fit}^{35}$ and individual model fits were not interpreted in light of this. Figure 1 depicts the general form of the APIM. Models were estimated using maximum likelihood estimation which allowed 
patients to be included even when caregiver information was missing. These individuals contributed information to the estimation of actor effects in the models. Separate models were fit for each combination of predictors and outcomes resulting in 9 models.

Results

Sample

Table 1 summarizes the demographic characteristics of the sample (patients $n=41$; caregivers $n=32$ ). Most patients were male $(70 \%, n=30)$. Caregivers were mostly female $(81 \%, n=26)$. Dyads primarily consisted of spousal pairs (65\%) or a close relative (e.g., adult child, $35 \%)$. There were no differences in predictors or outcomes based on the type of dyad (all p's > .18).

Generally, patients and caregivers scored below the cut off for adequate self-care on the individual HF self-care scales. With the exception of patient self-care maintenance, the scores for both groups fell below the standardized SCHFI score of 70 (Table 2$).^{28}$

To determine whether dyads with complete data differed from those with incomplete data, we conducted a missing data analysis by comparing scores for patients with available caregivers to those without. Our missing data analysis found that there were no significant differences for patients with or without available caregivers on the independent variables (all p's $>.28$ ). Specific to the HF self-care index, patients with available caregivers trended toward having lower HF self-care maintenance scores $(\mathrm{t}[38]=$ 2.00, $p=.053)$ and lower HF self-care self-efficacy $(t[38]=-1.88, p=.068)$. This suggests that patients with the greatest difficulty with self-care were more likely to have a caregiver who completed the questionnaire packet and returned it.

Patient and Caregiver Self-Report Outcomes

Means and standard deviations for all variables appear in Table 2. Using paired t-tests, we examined whether there were significant within-dyad differences. Depression was significantly different across 
members of the dyad with the patient reporting significantly more depressive symptoms (in the mild to moderate level) compared with the caregiver. HF self-care maintenance scores were also significantly higher (but just adequate) for the patient. All other comparisons were not significant.

\section{Relationships between Patient and Caregiver Self-Report Outcomes}

Tables 3 and 4 include the correlations for the variables of interest. The shaded diagonal in table 4 illustrates the level of agreement within a dyad for a given measure. Only a few correlations reached significance, most likely due to the small sample size, but the directions were consistent with previous theory and research. Higher levels of depression correlated with higher levels of anxiety and poorer perception of the relationship. Specific to the HF self-care index scales, patient perceptions of the relationship were significantly, positively related to a caregiver's rating of their self-efficacy. Additionally, caregiver depression was negatively correlated with patient HF self-care maintenance. The strongest correlation on the same variable was the dyad's perception of the relationship.

\section{Actor Partner Interdependence Models}

The goal of the current study was to explore the relationship of dyadic characteristics (e.g. anxiety, depression, and relationship quality) with HF self-care. Using the APIM framework, individual models were fit for each independent-dependent variable combination. Results for these models appear in Table 5 and are reported as standardized effects. Caregiver anxiety and depression each significantly predicted patient HF self-care maintenance as a partner effect. Higher levels of depression or anxiety for the caregiver predicted lower HF self-care maintenance scores for the patient. For the other HF self-care scales, only caregiver actor effects were significant. Higher caregiver anxiety predicted lower caregiver HF self-care management scores and higher caregiver ratings of relationship quality predicted greater caregiver ratings of self-efficacy.

\section{Discussion}


This study described the dyadic characteristics of mood and perception in HF patients and caregivers; then explored the relationship of the dyadic characteristics with HF self-care or how the dyad manages routine care and symptom exacerbations at home. What was found: 1) higher caregiver anxiety and depression was associated with lower patient engagement in self-care maintenance (treatment adherence and symptom monitoring); 2) higher caregiver anxiety was also associated with lower caregiver engagement in self-care management (symptom response and evaluation); and 3) caregiver perception of the relationship was associated with their self-efficacy in self-care.

Why is this study important? To date there has been little empiric evidence to support that caregivers have any effect on patient outcomes in HF. A recent study examined the association between family caregiver presence at clinic visits and self-care and suggested that caregiver satisfaction with clinician communication mediates the relationship between frequency of caregiver presence at the clinic visit and better HF self-care maintenance and management at home. ${ }^{36}$ Yet a review of clinical encounter videos conducted at the Mayo Clinic found that clinicians rarely engage caregivers, even when present. The clinician rarely assessed the reason for the caregivers presence at the visit; the caregiver's level of understanding of information provided; or his/her role in decision making. ${ }^{37}$ Taken together these studies highlight the importance of clinician interaction with the caregiver yet the linkage of caregivers to HF patient outcomes has been tenuous. In a recent systematic review of caregivers contributions to self-care only 2 out of 40 studies attempted to quantify this when social support and shared decision making were examined. ${ }^{7}$ This is the first study, to our knowledge, which explored the association of dyadic characteristics with HF self-care outcomes. Caregivers' mood states and perception of the relationship are associated with the patients' and their own engagement in self-care and caregiver self-efficacy. Given the small sample size, the statistical significance speaks to the strength of the signal.

Due to rigorous adherence and symptom monitoring needs, HF co-opts time and energy of patients and caregivers (if present). One way to tease out the impact of the caregiver in HF self-care is to examine 
partner effects in the patient's self-care. Partner effects in the APIM measure the contribution of the caregiver's independent variable to the patient's dependent variable accounting for the effect of the patient on their own variable. This study provides evidence that caregiver anxiety and depression are associated with poorer patient self-care maintenance. Simply put, the more anxious or depressed the caregiver was, the less likely that the patient was adherent or monitored their own symptoms. This finding is particularly intriguing as no actor effect of the patient's anxiety and depression on their own self-care maintenance was found despite the fact that the patients generally scored higher in depression (in the mild to moderate range) than the caregiver. This could be a function of our small sample size; with a larger sample we might have found patient effects, but it bears further consideration. Self-care maintenance involves everyday HF care. If the caregiver was anxious or depressed, the patient was less likely to report weighing themselves, checking their ankles for swelling, keeping clinician appointments, exercising, taking all of their medications, or watching their sodium intake. This caregiver effect on patient self-care maintenance may be a result of the anxious or depressed caregiver being less likely to cue the patient or that the patient is more concerned with the caregiver's state then with their own. Previously, strong, positive relationships were found in mixed (spousal, adult child, parent) dyad's shared care and self-care maintenance, supporting our findings that caregivers matter in self-care maintenance. ${ }^{38}$ What this study adds is that this engagement in self-care maintenance can be negatively impacted by caregivers' mood states.

The current health care system is built upon the assumption that caregivers will engage with the patient in self-care. But to what degree they are able to engage or what stressors in their own lives impact that engagement needs to be better understood. A way to tease out the correlates of caregiver engagement in HF self-care is to study actor effects. Actor effects measure the contribution of the caregiver's independent variable on the caregiver's dependent variable accounting for the effect of the patient. This study provides evidence that caregiver's anxiety is associated with their own engagement in self-care management and their perception of the relationship is associated with their overall self-efficacy for selfcare. The more anxious the caregiver, the poorer their engagement in making meaning of the patient's 
symptoms, taking action and then evaluating the response to the action taken. In addition, the higher the caregiver rates their satisfaction with their relationship with the patient, the higher their self-efficacy related to HF care. Self-efficacy is important as it has been found to mediate the relationship between social support and self-care management and moderate the relationship between self-care management and cost in $\mathrm{HF}^{8}$ In the same study that measured shared care, noted above, no associations were found between shared care with self-care management, however moderate to weak associations were found with self-care confidence. ${ }^{38}$ What this study adds is that caregivers are contributing to self-care management, however caregiver anxiety is associated with poorer contributions. A second finding is that the caregiver's perception of their relationship is related to their self-efficacy in self-care.

\section{Limitations}

This study has several limitations. First, the sample is primarily white and of moderate income. More diverse samples may result in different outcomes. Second, the sample is relatively small and measured at one time point. Once again, a larger sample measured over time may provide additional findings.

Implications for Outpatient Palliative Care

There are several implications for palliative care found in this study. First, these findings may have relevance to other chronic disease populations with rigorous self-care requirements. Clinicians who automatically assume that caregivers will supplement patient self-care may want to give added thought to this assumption. Caregivers may or may not have their own chronic illnesses and suffer from poor selfefficacy impacting their ability to engage. Some of these stressors, such as anxiety and depression can be mitigated, potentially resulting in improved patient self-care. However, in the present health care system, providers are not reimbursed for providing family interventions; thus, care for caregivers is unlikely to happen. Secondly, assessing for these stressors and providing additional self-care information involves communication skills. Clinicians need to talk to the patient and caregiver about their mood states and their relationship, particularly in dyads with a history of poor self-care. As noted earlier caregiver satisfaction with 
clinician communication mediates the relationship between frequency of caregiver presence at the clinic visit and better HF self-care maintenance and management at home. Communication is a non-pharmacological intervention that improves patient outcomes.

\section{Conclusion}

In summary, our study was designed to answer some of the questions related to caregiver's impact on self-care outcomes in HF. We selected three modifiable independent variables - anxiety, depression, and perception of the relationship to first describe the dyadic characteristics in HF patients and caregivers, then explore the relationship of these dyadic characteristics with HF self-care. What we found was that caregivers' mood states and perception of the relationship impacts the patient and their own engagement in HF self-care as well as the caregiver's self-efficacy. The next steps in this line inquiry is to determine if dyadic HF self-care interventions which address mood states and support the relationship result in better self-care outcomes.

Acknowledgements: This study was supported by the National Palliative Care Research Center Junior Faculty Career Development Award.

Author Disclosure Statement: No competing financial interests exist. 
References

1. Yancy CW, Jessup M, Bozkurt B, et al. 2013 ACCF/AHA Guideline for the Management of Heart Failure: Executive Summary: A Report of the American College of Cardiology Foundation/American Heart Association Task Force on Practice Guidelines. Journal of the American College of Cardiology. 2013;62:1495-1539.

2. Mozaffarian D, Benjamin EJ, Go AS, et al. Heart Disease and Stroke Statistics-2015 Update: A Report From the American Heart Association. Circulation. 2014.

3. Adler ED, Goldfinger JZ, Kalman J, Park ME, Meier DE. Palliative care in the treatment of advanced heart failure. Circulation. 2009;120:2597-2606.

4. Cook C, Cole G, Asaria P, Jabbour R, Francis DP. The annual global economic burden of heart failure. International Journal of Cardiology. 2013.

5. Heidenreich PA, Albert NM, Allen LA, et al. Forecasting the Impact of Heart Failure in the United States: A Policy Statement From the American Heart Association. Circulation: Heart Failure. 2013.

6. Clark AM, Spaling M, Harkness K, et al. Determinants of effective heart failure self-care: a systematic review of patients' and caregivers' perceptions. Heart. 2014.

7. Buck HG, Harkness K, Wion R, et al. Caregivers' contributions to heart failure self-care: A systematic review. European journal of cardiovascular nursing : journal of the Working Group on Cardiovascular Nursing of the European Society of Cardiology. 2014.

8. Riegel B, Dickson VV. A situation-specific theory of heart failure self-care. Journal of Cardiovascular Nursing. 2008;23:190-196.

9. Riegel B, Lee CS, Dickson VV. Self care in patients with chronic heart failure. Nat Rev Cardiol. 2012;8:644-654.

10. Vellone E, Chung ML, Cocchieri A, Rocco G, Alvaro R, Riegel B. Effects of Self-Care on Quality of Life in Adults with Heart Failure and Their Spousal Caregivers: Testing Dyadic Dynamics Using the Actor-Partner Interdependence Model. Journal of Family Nursing. 2014;20:120-141.

11. Lee CS, Vellone $\mathrm{E}$, Lyons $\mathrm{KS}$, et al. Patterns and predictors of patient and caregiver engagement in heart failure care: a multi-level dyadic study. International Journal of Nursing Studies. 2014.

12. Cook WL, Kenny DA. The Actor-Partner Interdependence Model: A model of bidirectional effects in developmental studies. International Journal of Behavioral Development. 2005;29:101-109.

13. Chung ML, Moser DK, Lennie TA, Rayens MK. The effects of depressive symptoms and anxiety on quality of life in patients with heart failure and their spouses: Testing dyadic dynamics using Actor-Partner Interdependence Model. Journal of Psychosomatic Research. 2009;67:29-35.

14. Rohrbaugh MJ, Shoham V, Cleary AA, Berman JS, Ewy GA. Health consequences of partner distress in couples coping with heart failure. Heart \&amp; Lung: The Journal of Acute and Critical Care. 2009;38:298-305.

15. Jurgens CY. Somatic awareness, uncertainty, and delay in care-seeking in acute heart failure. Research in Nursing and Health. 2006;29:74-86.

16. Sebern M. Shared Care, elder and family member skills used to manage burden. Journal of advanced nursing. 2005;52:170-179.

17. Berg CA, Upchurch R. A developmental-contextual model of couples coping with chronic illness across the adult life span. Psychol Bull. 2007;133:920-954.

18. Hoppmann C, Gerstorf D. Spousal Interrelations in Old Age: A Mini-Review. Gerontology. 2009;55:449-459.

19. Bekelman DB, Nowels CT, Retrum JH, et al. Giving voice to patients' and family caregivers' needs in chronic heart failure: implications for palliative care programs. Journal of palliative medicine. 2011;14:1317-1324. 
20. Retrum JH, Nowels CT, Bekelman DB. Patient and caregiver congruence: The importance of dyads in heart failure care. Journal of Cardiovascular Nursing. 2013;28:129-136.

21. Lum HD, Lo D, Hooker S, Bekelman DB. Caregiving in heart failure: Relationship quality is associated with caregiver benefit finding and caregiver burden. Heart \& Lung: The Journal of Acute and Critical Care. 2014;43:306-310.

22. Kenny DA, Kashy DA, Cook WL. Dyadic data analysis. Methodology in the social sciences; Variation: Methodology in the social sciences.: Guilford Press; 2006.

23. Kitko LA, Hupcey JE, Gilchrist JH, Boehmer JP. Caring for a spouse with end-stage heart failure through implantation of a left ventricular assist device as destination therapy. Heart \& Lung: The Journal of Acute and Critical Care. 2013;42:195-201.

24. Kroenke K, Spitzer RL, Williams JB. The PHQ-9: validity of a brief depression severity measure. J Gen Intern Med. 2001;16:606-613.

25. Derogatis LR, Melisaratos N. The Brief Symptom Inventory: an introductory report. Psychological medicine. 1983;13:595-605.

26. Sharpley CF, Rogers HJ. Preliminary Validation of the Abbreviated Spanier Dyadic Adjustment Scale: Some Psychometric Data Regarding a Screening Test of Marital Adjustment. Educational and Psychological Measurement. 1984;44:1045-1049.

27. Spanier GB. Measuring Dyadic Adjustment: New Scales for Assessing the Quality of Marriage and Similar Dyads. Journal of Marriage and Family. 1976;38:15-28.

28. Riegel B, Lee CS, Dickson VV, Carlson B. An update on the self-care of heart failure index. Journal of Cardiovascular Nursing. 2009;24:485-497.

29. Riegel B, Carlson B, Moser DK, Sebern M, Hicks FD, Roland V. Psychometric testing of the selfcare of heart failure index. Journal of Cardiac Failure. 2004;10:350-360.

30. Riegel B, Carlson B, Glaser D. Development and testing of a clinical tool measuring selfmanagement of heart failure. Heart Lung. 2000;29:4-15.

31. Vellone E, Riegel, B., Cocchieri, A., Barbaranelli, C., D'Agostino, F., Glaser, D., Rocco, G., and Alvaro, R. Validity and Reliability of the Caregiver Contribution to Self-care of Heart Failure Index. Journal of cardiovascular nursing. 2013;28:245-255.

32. Vellone E, D'Agostino F, Buck HG, et al. The key role of caregiver confidence in the caregiver's contribution to self-care in adults with heart failure. European journal of cardiovascular nursing : journal of the Working Group on Cardiovascular Nursing of the European Society of Cardiology. 2014.

33. Kashy DA, Kenny, D.A. The analysis of data from dyads and groups. In: Reis HTJCM, ed. Handbook of research methods in social and personality psychology: Cambridge University Press; 2000.

34. Muthén LK, \& Muthén, B. O. Mplus user's guide : statistical analysis with Laten variables. Los Angeles, CA: Muthén \& Muthén.; 2007.

35. Kline RB. Principles and Practice of Structural Equation Modeling. New York: The Guilford Press; 1998.

36. Cené CW, Haymore LB, Lin F-C, et al. Family member accompaniment to routine medical visits is associated with better self-care in heart failure patients. Chronic Illness. 2014.

37. Boehmer KR, Egginton JS, Branda ME, et al. Missed opportunity? Caregiver participation in the clinical encounter. A videographic analysis. Patient education and counseling. 2014.

38. Sebern M, Riegel B. Contributions of supportive relationships to heart failure self-care. European Journal of Cardiovascular Nursing. 2009;8:97-104. 
Figure 1. Diagram of Actor-Partner Interdependence model

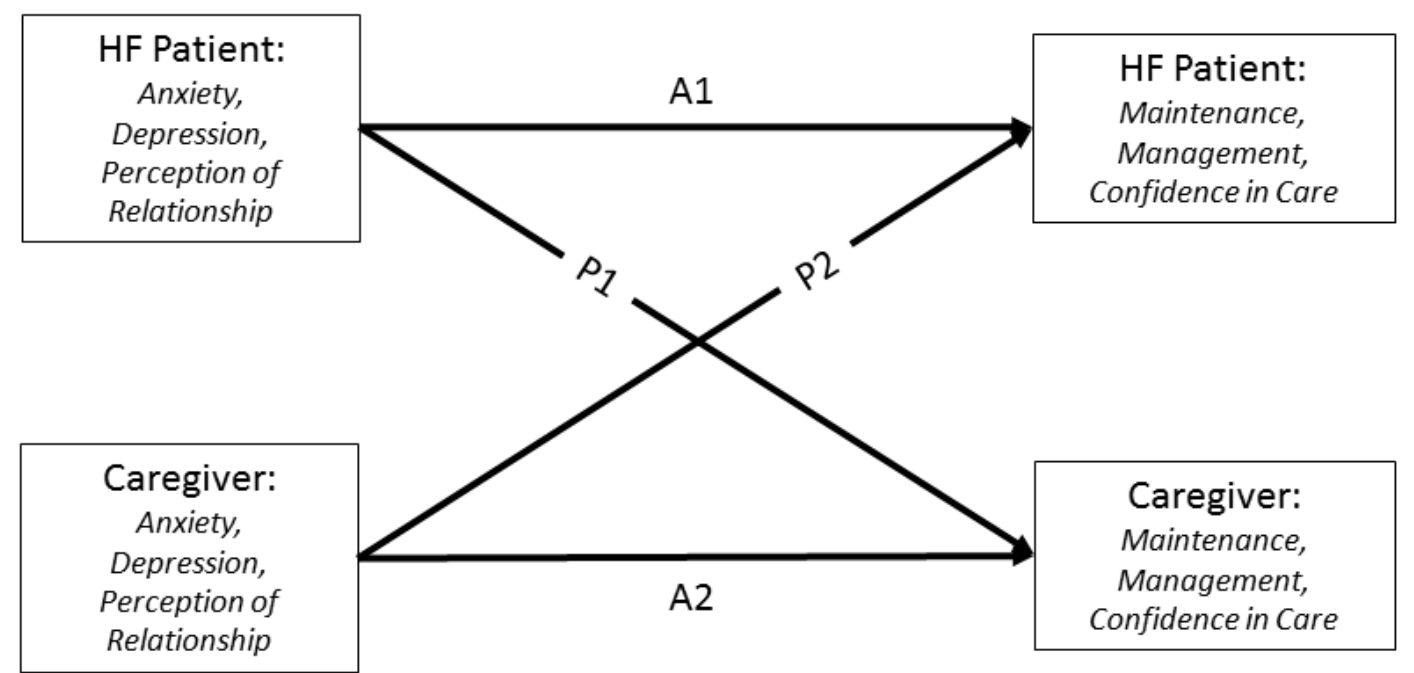

Note. Errors terms were estimated and patient and caregiver predictors and outcomes were allowed to covary but are omitted from the diagram. 
Table 1. Sample Demographic Characteristics

\begin{tabular}{lcc}
\multicolumn{1}{c}{ Characteristics } & Patient (n=41) & Caregiver (n=32) \\
\hline Age (in years) & Mean (SD) or proportion & Mean (SD) or proportion \\
\hline Gender (\% Female) & $71.19(12.45)$ & $58.84(16.20)$ \\
\hline Race/Ethnicity & $30.2 \%$ & $81.3 \%$ \\
White & & \\
Black & $80.9 \%$ & $66 \%$ \\
\hline Education & $6.4 \%$ & $32 \%$ \\
HS or less & & \\
Some college & $53.7 \%$ & $50 \%$ \\
College or & $22 \%$ & $25 \%$ \\
advanced degree & $24.3 \%$ & $25 \%$ \\
\hline Income to make ends meet & & \\
More than enough & & \\
Enough & $17.5 \%$ & $25 \%$ \\
Not enough & $52.5 \%$ & $59.4 \%$ \\
\hline
\end{tabular}


Table 2. Patient and Caregiver Self-Report Means and Standard Deviations

\begin{tabular}{|c|c|c|c|c|c|c|c|c|}
\hline & \multicolumn{3}{|c|}{ Patient } & \multicolumn{3}{|c|}{ Caregiver } & \multicolumn{2}{|c|}{$\begin{array}{c}\text { Test for } \\
\text { differences }\end{array}$} \\
\hline & Mean & $\mathrm{N}$ & SD & Mean & $\mathrm{N}$ & SD & $t$ & p-value \\
\hline Anxiety & 0.57 & 40 & 0.68 & 0.59 & 31 & 0.76 & -0.24 & 0.809 \\
\hline Depression & 8.83 & 40 & 5.54 & 4.13 & 31 & 5.23 & 3.01 & 0.005 \\
\hline $\begin{array}{l}\text { Perception of } \\
\text { Relationship }\end{array}$ & 23.25 & 40 & 5.07 & 24.29 & 31 & 4.75 & -0.8 & 0.432 \\
\hline Maintenance & 70.10 & 40 & 14.24 & 54.99 & 29 & 17.01 & 3.37 & 0.002 \\
\hline Management & 56.42 & 36 & 25.94 & 57.41 & 24 & 20.37 & -0.44 & 0.663 \\
\hline Care Self-efficacy & 55.33 & 40 & 19.90 & 52.37 & 30 & 22.14 & -0.18 & 0.855 \\
\hline
\end{tabular}


Table 3. Within-patient and within caregiver correlations

\begin{tabular}{|c|c|c|c|c|c|}
\hline \multicolumn{6}{|c|}{ Patient Correlations } \\
\hline & Anxiety & Depression & $\begin{array}{l}\text { Perception of } \\
\text { Relationship }\end{array}$ & Maintenance & Management \\
\hline Depression & $.60^{*}$ & & & & \\
\hline $\begin{array}{l}\text { Perception of } \\
\text { Relationship }\end{array}$ & -.13 & $-.30+$ & & & \\
\hline Maintenance & .14 & .09 & .07 & & \\
\hline Management & .04 & .00 & -.01 & $.40^{*}$ & \\
\hline $\begin{array}{l}\text { Care Self- } \\
\text { Efficacy }\end{array}$ & .04 & .03 & .25 & .14 & $.37^{*}$ \\
\hline
\end{tabular}

\section{Caregiver Correlations}

\begin{tabular}{|c|c|c|c|c|c|}
\hline & Anxiety & Depression & $\begin{array}{l}\text { Perception of } \\
\text { Relationship }\end{array}$ & Maintenance & Management \\
\hline Depression & $.82 *$ & & & 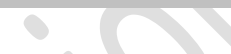 & \\
\hline $\begin{array}{l}\text { Perception of } \\
\text { Relationship }\end{array}$ & $-.42 *$ & $-.56 *$ & & & \\
\hline Maintenance & -.24 & -.19 & .24 & $(3)$ & \\
\hline Management & -.27 & -.21 & .15 & .37 & \\
\hline $\begin{array}{l}\text { Care Self- } \\
\text { Efficacy }\end{array}$ & -.20 & -.23 & $.60^{*}$ & .25 & $.42 *$ \\
\hline
\end{tabular}

Note. ${ }^{*} p<.05,+p<.07$ 
Table 4. Relationships between Patient and Caregiver Self-Report Outcomes

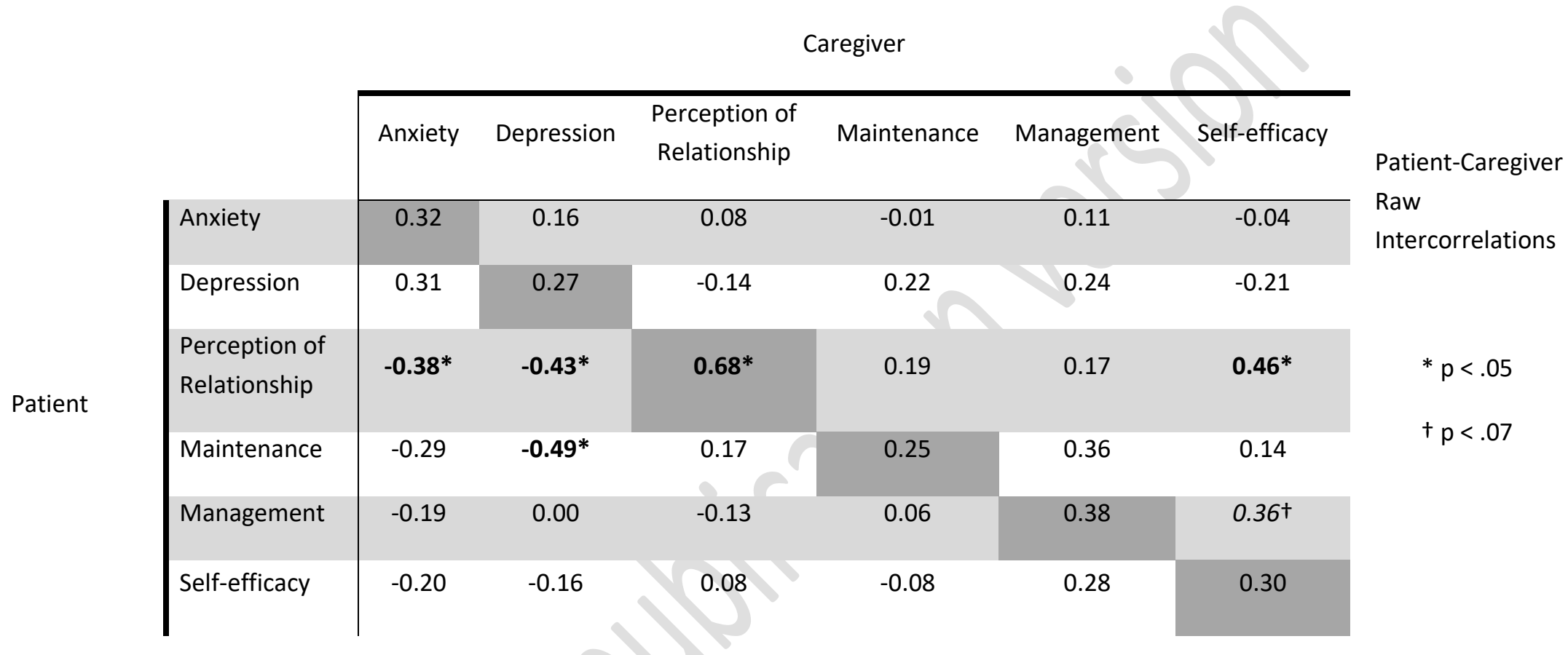


Table 5. Actor Partner Interdependence Models

Outcome:

\begin{tabular}{|c|c|c|c|c|c|c|c|c|c|}
\hline & \multicolumn{3}{|c|}{ Maintenance } & \multicolumn{3}{|c|}{ Management } & \multicolumn{3}{|c|}{ Self-efficacy } \\
\hline Anxiety & $b$ & $S E$ & $p$-value & $b$ & $S E$ & $p$-value & $b$ & $S E$ & $p$-value \\
\hline Patient: Actor (A1) & 0.29 & 0.15 & 0.06 & 0.11 & 0.17 & 0.52 & 0.11 & 0.16 & 0.51 \\
\hline Patient: Partner (P1) & 0.05 & 0.17 & 0.76 & 0.15 & 0.17 & 0.34 & 0.03 & 0.17 & 0.87 \\
\hline Caregiver: Actor (A2) & -0.27 & 0.18 & 0.14 & -0.38 & 0.18 & 0.04 & -0.21 & 0.18 & 0.25 \\
\hline Caregiver: Partner (P2) & -0.43 & 0.16 & 0.01 & -0.22 & 0.18 & 0.23 & -0.24 & 0.19 & 0.21 \\
\hline Depression & $b$ & $S E$ & $p$-value & $b$ & $S E$ & $p$-value & $b$ & $S E$ & $p$-value \\
\hline Patient: Actor (A1) & 0.24 & 0.14 & 0.09 & 0.02 & 0.18 & 0.91 & 0.07 & 0.16 & 0.66 \\
\hline Patient: Partner (P1) & 0.26 & 0.16 & 0.12 & 0.29 & 0.18 & 0.10 & -0.12 & 0.17 & 0.50 \\
\hline Caregiver: Actor (A2) & -0.26 & 0.17 & 0.13 & -0.30 & 0.17 & 0.07 & -0.19 & 0.18 & 0.28 \\
\hline Caregiver: Partner (P2) & -0.56 & 0.13 & & -0.02 & 0.19 & 0.93 & -0.16 & 0.19 & 0.41 \\
\hline Perception of Relationship & $b$ & & $p$-value & $b$ & $S E$ & $p$-value & $b$ & $S E$ & $p$-value \\
\hline Patient: Actor (A1) & 0.02 & 0.23 & 0.93 & 0.16 & 0.24 & 0.50 & 0.39 & 0.22 & 0.08 \\
\hline Patient: Partner (P1) & 0.01 & 0.24 & 0.97 & 0.18 & 0.29 & 0.53 & 0.09 & 0.20 & 0.65 \\
\hline Caregiver: Actor (A2) & 0.23 & 0.24 & 0.35 & -0.02 & 0.29 & 0.94 & 0.54 & 0.18 & $<.01$ \\
\hline Caregiver: Partner (P2) & 0.08 & 0.25 & 0.76 & -0.31 & 0.25 & 0.22 & -0.20 & 0.25 & 0.42 \\
\hline
\end{tabular}

Note. All $b$ s are standardized using XY standardization. 